\title{
Antitumor potential of a novel camptothecin derivative, $\mathrm{ZBH}-\mathrm{ZM}-06$
}

\author{
DI WU ${ }^{1}$, DA-WEI ZHAO ${ }^{2}$, YONG-QI LI ${ }^{1}$, WEI-GUO SHI ${ }^{3}$, QI-LIANG YIN ${ }^{1}$, ZHENG-KUN TU $^{4}$, \\ YING-YING YU ${ }^{1}$, BO-HUA ZHONG ${ }^{3}, \mathrm{HONG} \mathrm{YU}^{5}$ and WAN-GUO BAO ${ }^{6}$
}

\begin{abstract}
${ }^{1}$ Cancer Centre, The First Hospital of Jilin University, Changchun, Jilin $130021 ;{ }^{2}$ Department of Breast Tumor, Jilin Province Tumor Hospital, Changchun, Jilin 130012; ${ }^{3}$ Institute of Pharmacology and Toxicology Academy of Military Medical Sciences, Beijing 100850; ${ }^{4}$ Institute of Translational Medicine, The First Hospital of Jilin University, Changchun, Jilin 130061; ${ }^{5}$ Cell Biology Laboratory, Jilin Province Institute of Cancer Prevention and Treatment, Jilin Cancer Hospital;

${ }^{6}$ Department of Infectious Disease, The First Hospital of Jilin University, Changchun, Jilin 130021, P.R. China
\end{abstract}

Received June 15, 2017; Accepted December 4, 2017

DOI: 10.3892/or.2017.6143

\begin{abstract}
Camptothecin (CPT) is a cytotoxic quinoline alkaloid that is used clinically as an anticancer drug. However, the clinical application of CPT is limited due to its low solubility as well as serious and unfathomable side-effects. In the present study, we created a novel 10-hydroxy CPT prodrug, ZBH-ZM-06. Its cellular cytotoxic activity was analyzed in terms of cellular viability, acetylcholinesterase (AchE) inhibition, DNA relaxation, cellular cycling and apoptosis properties. Our results showed that the AchE inhibition rate of $10 \mu \mathrm{mol} / 1$ ZBH-ZM-06 was $12.5 \%$, compared to $96.5 \%$ for carbonyloxycamptothecin (CPT-11). In a chemical stability assay, only $4.9 \%$ of $\mathrm{ZBH}-\mathrm{ZM}-06$ remained after $4 \mathrm{~h}$ at $\mathrm{pH}$ 7.4. In addition, $10 \mu \mathrm{mol} / 1 \mathrm{ZBH}-\mathrm{ZM}-06$ significantly inhibited the tumor cell viability of nine tumor cell lines, compared to CPT-11 and the CPT active ingredient, 7-ethyl-10-hydroxy-camptothecin (SN38) $(\mathrm{P}<0.01-0.05)$. In the apoptosis assay, ZBH-ZM-06 increased the ratio of Annexin $\mathrm{V}^{+} /$propidium iodide $(\mathrm{PI})^{-/ 4}$ cells by flow cytometric analysis $(\mathrm{P}<0.05)$. Moreover, ZBH-ZM-06 activated caspase- 3 and poly(ADP-ribose)polymerase (PARP) expression by immunoblotting. Furthermore, ZBH-ZM-06 induced a greater $\mathrm{G}_{2} / \mathrm{M}$ phase arrest ratio, compared to CPT-11 and SN38. These results indicated that ZBH-ZM-06 had higher antitumor activity than CPT-11 and SN38, which was
\end{abstract}

Correspondence to: Dr Hong Yu, Cell Biology Laboratory, Jilin Province Institute of Cancer Prevention and Treatment, Jilin Cancer Hospital, 1018 Huguang Road, Changchun, Jilin 130021, P.R. China E-mail: yhzhlyy@126.com

Dr Wan-Guo Bao, Department of Infectious Disease, The First Hospital of Jilin University, 71 Xinmin Road, Changchun, Jilin 130021, P.R. China

E-mail: baowanguo1963@aliyun.com

Key words: camptothecin derivative, antitumor, topoisomerase, proapoptosis, cell cycle shown by its: i) release of the effective ingredient; ii) growth inhibition of a broad spectrum of tumor cells; iii) inhibition of DNA topoisomerase (Topo-1); and iv) promotion of apoptosis through an intrinsic signaling pathway. Thus, ZBH-ZM-06 may be applied in the preclinic study for cancer treatment.

\section{Introduction}

Camptothecin (CPT) is a cytotoxic quinoline alkaloid isolated from Camptotheca acuminta, a type of tree natively growing in China, which was discovered in the 1960s (1). The antitumor activity of CPT depends on a highly specific inhibition of Topo-1. This activity is achieved by docking at the enzymeDNA interface to stabilize the formation of Topo-1-DNA cleavable complexes, thus prohibiting DNA strand religation. Once the stable form of the cleavable complex is broken by some mechanism, such as replication or transcription caused by some cytotoxic drugs, the breakage of the Topo-1-linked DNA single-strand can cause DNA double-strand breaks (DSBs) (2). Subsequently, these DSBs will trigger a DNA damage response to activate serine-threonine kinases to drive the ATM-CHK2- and ATR-CHK1-mediated checkpoint pathways as well as $\mathrm{H} 2 \mathrm{AX}$ phosphorylation to arrest the cell cycle at the $G_{1} / S$ and $G_{2} / M$ phases (3). The clinical application of CPT is limited due to its low solubility as well as serious and unfathomable side-effects $(4,5)$. To overcome these drawbacks, several CPT derivatives have been developed to date, including topotecan (9-dimethyl amino-10-hydroxy camptothecin; TPT) and irinotecan (7-ethyl-10-[4-(1-piperidino)-1-piperidino] carbonyloxycamptothecin; CPT-11) (6,7). The US Food and Drug Administration has approved these CPT derivatives for ovarian and colon cancer treatment $(8,9)$. Furthermore, their anticancer potential on other tumors also has been reported, such as lung $(7,10)$, breast (11), pancreatic cancer (12), lymphoma (13), glioma (14) and leukemia (15).

SN38, the prodrug of CPT-11, is approximately 100- to 1,000-fold more cytotoxic than CPT-11 (16). CPT-11 plays an antitumor role in vivo through the release of its active form SN38 by liver carboxylesterase. However, there are significant individual differences among the antitumor activity of 
CPT-11 due to its low enzymatic conversion rate in vivo and its uncertain pharmacokinetic properties among individuals. In addition, the CPT-11 prodrug group (4-piperidinyl piperidine) causes AchE inhibition, which easily leads to acetylcholine syndrome, resulting in early severe diarrhea and other sideeffects (10).

In order to overcome the above drawbacks of CPT-11, scientists have made several attempts to improve it (10,17-19). In the present study, we designed and synthesized a novel 10-hydroxy CPT prodrug with a high efficiency and low toxicity $(20,21)$. Its cytotoxic activity, side-effects, antitumor activity and possible mechanism were analyzed in multiple assays. The results demonstrated that ZBH-ZM-06 has optimal antitumor properties and fewer side-effects than those of CPT-11 and SN38.

\section{Materials and methods}

Derivative of CPT. The new 10-hydroxy CPT prodrug, ZBH-ZM-06, was designed and synthesized by the Institute of Pharmacology and Toxicology Academy of Military Medical Sciences (20). By using a linear amino acid as a linker, the $\mathrm{N}$-terminal amino acid was ligated with $10-\mathrm{OH}$ of SN38 through a urethane bond. Next, through removal of the benzyl protecting group by catalytic hydrogenation, a carboxyl group was liberated. The selected $N$-methylpiperazine, which has good biocompatibility, was conjugated with the free carboxyl group of the amino acid through an amide bond. The basic nitrogen atom of $\mathrm{N}$-methylpiperazine can form a salt with the carboxyl group to improve the water solubility of the compound.

CPT-11 and SN38 were also provided by the Institute of Pharmacology and Toxicology Academy of Military Medical Sciences. All of the compounds were dissolved in dimethyl sulfoxide (DMSO; Sigma-Aldrich, St. Louis, MO, USA) at $10 \mathrm{mmol} / \mathrm{l}$ as a stock reagent. Further dilutions were made with Iscove's modified Dulbecco's medium (IMDM; Life Technologies, Grand Island, NY, USA) at the appropriate concentrations and stored at $-20^{\circ} \mathrm{C}$.

AchE inhibition assay and stability test. The AchE inhibition test by ZBH-ZM-06 was performed as previously described (20). The stability of ZBH-ZM-06 was analyzed at 1 , 2, 4, 8 and 12 h in phosphate-buffered saline (PBS; pH 7.4 and pH 5.0) by high-performance liquid chromatography (HPLC) with a C18 analytical column, as previously described (20).

Cell culture and cytotoxicity assay. Twelve human tumor cell lines, including SW1116, SAOS-2, A549, SGC-7901, 7860, HeLa, SK-OV-3, K562, NCI-H446, A375, MCF-7, SMMC-7721 and NCM460, a normal human colon mucosal epithelial cell line, were incubated with IMDM or RPMI-1640 medium (Gibco, Carlsbad, CA, USA) supplemented with $10 \%$ fetal calf serum (FCS), $100 \mathrm{U} / \mathrm{ml}$ penicillin, $2 \mathrm{mmol} / 1$ glutamine, and $100 \mu \mathrm{g} / \mathrm{ml}$ streptomycin at $37^{\circ} \mathrm{C}$ in a humidified atmosphere incubator containing $5 \% \mathrm{CO}_{2}$. Cells were harvested during the logarithmic growth phase and plated in 96-well plates at $2.5 \times 10^{3}$ cells/well in $100 \mu \mathrm{l}$ of medium. After incubation for $24 \mathrm{~h}, 0.0032 \mu \mathrm{mol} / 1$ to $50 \mu \mathrm{mol} / 1 \mathrm{ZBH}-\mathrm{ZM}-06$, CPT-11 or SN38 was added to the indicated plate. The cellular viability was determined by the 3-(4,5-dimethylthiazol-2-yl)2,5-diphenyltetrazolium bromide (MTT) assay $72 \mathrm{~h}$ later (22). The absorbance at $490 \mathrm{~nm}$ was detected by a microplate reader. The half-maximal inhibition concentration $\left(\mathrm{IC}_{50}\right)$ values of the analyzed drugs were calculated. Each experiment was repeated at least three times.

DNA relaxation assay. To evaluate the effects of $\mathrm{ZBH}-\mathrm{ZM}-06$ and CPT-11 on DNA relaxation, the TopoGEN Topoisomerase 1 Drug Screening kit (TopoGEN, Inc., Port Orange, FL, USA) was employed, according to the manufacturer's instructions. Closed loop superhelix plasmid DNA (pHOT1) was co-incubated with recombinant wild-type human Topo-1 (2 U, TG2005H-RC1; TopoGEN) at $37^{\circ} \mathrm{C}$ for $30 \mathrm{~min}$ in the presence or absence of the drugs in Topo-1 reaction buffer. Reactions were quenched by incubation with sodium dodecyl sulfate (1\%) and proteinase $\mathrm{K}(50 \mu \mathrm{g} / \mathrm{ml})$ for $15 \mathrm{~min}$ at $37^{\circ} \mathrm{C}$. The DNA samples were then analyzed by electrophorese on $1 \%$ agarose gel containing $1 \mu \mathrm{g} / \mathrm{ml}$ ethidium bromide. Gels were visualized and photographed under ultraviolet light. Each experiment was performed in triplicate.

Cell cycle analysis. K562, SK-OV-3 and SW1116 cells were seeded in 6-well plates at 5x10\% $/$ well in FCS-free IMDM. After incubation for $24 \mathrm{~h}$, the cells were exposed to ZBH-ZM-06, CPT-11, or SN38 at $10 \mu \mathrm{mol} / 1$ in IMDM containing $10 \%$ FCS and harvested after 24,48 and $72 \mathrm{~h}$, respectively. The cells were stained with the Coulter DNA PREP ${ }^{\mathrm{TM}}$ reagents kit (Beckman Coulter, Brea, CA, USA), according to the manufacturer's protocol. The cell cycle was analyzed by flow cytometry using a Coulter EPICS XL-MCL instrument. Data were analyzed using MultiCycle 32-bit Version Software (Phoenix Flow Systems, San Diego, CA, USA).

Measurement of tumor cell apoptosis. The FITC Annexin V Apoptosis Detection kit I (BD Biosciences, San Diego, CA, USA) and the FITC Active Caspase-3 Apoptosis kit (550480; BD Biosciences) were employed for the measurement of cell apoptosis. K562, SK-OV-3 and SW1116 cells $\left(5 \times 10^{5}\right)$ were seeded on 6-well plates and cultured overnight. The next day, $10 \mu \mathrm{mol} / \mathrm{l} \mathrm{ZBH}-\mathrm{ZM}-06, \mathrm{CPT}-11$, or SN38 was added for the indicated time $(24,48$ and $72 \mathrm{~h})$. After treatment, the cells were harvested, washed and resuspended in $100 \mu \mathrm{l}$ of binding buffer containing $5 \mu \mathrm{l}$ of Annexin V-FITC and $5 \mu \mathrm{l}$ of propidium iodide (PI), or stained with FITC-conjugated cleaved caspase-3 antibody for $15-20 \mathrm{~min}$ in the dark at $20^{\circ} \mathrm{C}$. Next, viable, apoptotic, necrotic cells, and activated caspase $-3^{+}$cells were analyzed by an Epics XL/MCL flow cytometer.

Western blot analysis. SW1116 cells were incubated with $10 \mu \mathrm{mol} / 1 \mathrm{ZBH}-\mathrm{ZM}-06$, CPT-11, or SN38 for $72 \mathrm{~h}$. The cell lysate preparation and immunoblot analysis were performed as previously described (21).

Statistical analysis. Experimental data were analyzed by the t-test, one-way analysis of variance, and chi-squared test with SPSS 23.0 software (version 23.0; SPSS, Inc., Chicago, IL, USA). Data were expressed as the mean \pm standard deviation (SD) using a minimum of triplicate determinations. $\mathrm{P}<0.05$ was considered to be statistically significant. 

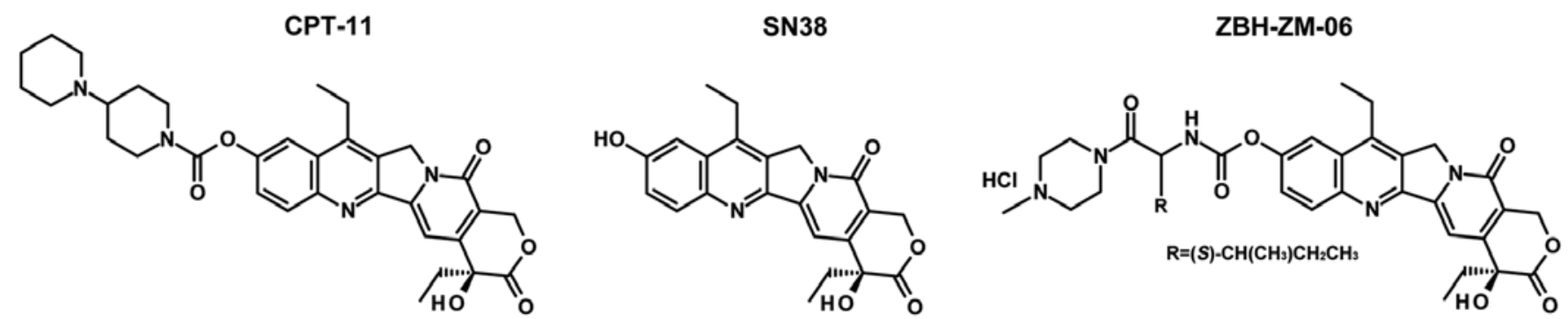

Figure 1. Molecular structures of CPT-11, SN38 and ZBH-ZM-06. ZBH-ZM-06 was constructed by: i) coupling of the 10-OH substituent of SN38 and an N-terminal amino acid via a urethane bond; ii) removal of the benzyl protecting group by catalytic hydrogenation to release a carboxyl group; and iii) conjugation of $N$-methylpiperazine with the free carboxyl group of an amino acid by an amide bond. The basic nitrogen atom of $N$-methylpiperazine can form a salt with the carboxyl group to improve the water solubility of the compound.

Table I. The AchE inhibitory activity of ZBH-ZM-06 and CPT-11.

\begin{tabular}{lrrrc}
\hline & \multicolumn{4}{c}{ Inhibition (\%) } \\
\cline { 2 - 5 } & $0.1 \mu \mathrm{M}$ & $1 \mu \mathrm{M}$ & $10 \mu \mathrm{M}$ & $100 \mu \mathrm{M}$ \\
\hline ZBH-ZM-06 & 4.9 & 8.4 & 12.5 & 44.2 \\
CPT-11 & 45.7 & 78.9 & 96.5 & - \\
\hline
\end{tabular}

Table II. The chemical stability of ZBH-ZM-06 as indicated by the percentage of remaining compound after incubation for various times and $\mathrm{pH}$ values.

\begin{tabular}{ccccccc}
\hline & $0 \mathrm{~h}$ & $1 \mathrm{~h}$ & $2 \mathrm{~h}$ & $4 \mathrm{~h}$ & $8 \mathrm{~h}$ & $12 \mathrm{~h}$ \\
\hline $\mathrm{pH} \mathrm{7.4}$ & 98.1 & 38.8 & 17.7 & 4.9 & - & - \\
pH 5.0 & 99.8 & - & - & 97.8 & 95.0 & 92.8 \\
\hline
\end{tabular}

\section{Results}

Decreased AchE inhibitory activity of ZBH-ZM-06. The molecular structures of CPT-11, SN38 and ZBH-ZM-06 are shown in Fig. 1. CPT-11 is a potent inhibitor of AchE and can cause acute cholinergic diarrhea. In order to test whether ZBH-ZM-06 causes this side-effect, the inhibitory activity of ZBH-ZM-06 on AchE was detected. Obviously, ZBH-ZM-06 revealed a lower potential to inhibit AchE compared with CPT-11 at different concentrations (Table I). This result indicates that the toxic side-effect of AchE inhibition of $\mathrm{ZBH}-\mathrm{ZM}-06$ is less than that of CPT-11.

ZBH-ZM-06 can fully release the active ingredient $S N 38$ under physiological pH conditions. The stability of ZBH-ZM-06 was investigated in phosphate-buffered saline (PBS) by HPLC using a C18 analytical column. The mobile phase consisted of water and acetonitrile at a ratio of 70:30, respectively, containing $1 \%$ trifluoroacetic acid. ZBH-ZM-06 and its metabolite SN38 were separated at a flow rate of $0.8 \mathrm{ml} / \mathrm{min}$ and detected at a wavelength of $370 \mathrm{~nm}$. ZBH-ZM-06 was dissolved in PBS $(0.2 \mathrm{mg} / \mathrm{ml}, \mathrm{pH} 7.4$ and $\mathrm{pH} 5.0)$, incubated at $37^{\circ} \mathrm{C}$ and analyzed at $0,1,2,4,8$ and $12 \mathrm{~h}(20)$. The results are summarized in Table II. ZBH-ZM-06 showed a pH-dependent stability. ZBH-ZM-06 was relatively stable, as shown by the finding that $92.8 \%$ still remained after $12 \mathrm{~h}$ at $\mathrm{pH} 5.0,37^{\circ} \mathrm{C}$. However, at $\mathrm{pH} 7.4$, only $4.9 \%$ remained after incubation for $4 \mathrm{~h}$. The antitumor activity of CPT-11 is achieved through the release of its active ingredient, SN38 (23), by liver carboxylesterase. Nevertheless, there are significant individual differences in the antitumor activity of CPT-11 due to its low enzymatic conversion rate in vivo and its uncertain pharmacokinetic properties among individuals. ZBH-ZM-06 can fully release the active ingredient, $\mathrm{SN} 38$, under physiological $\mathrm{pH}$ ( $\mathrm{pH}$ 7.4); therefore, this non-liver release-dependent property may improve the drug efficacy.

Inhibition of tumor cell viability by $\mathrm{ZBH}-\mathrm{ZM}-06$. The inhibitory activity of ZBH-ZM-06 on cellular viability was detected by MTT assay in 12 tumor cell lines. The NCM460 cell line was used as a normal cell line control. The $\mathrm{IC}_{50}$ values are summarized in Table III. The most sensitive cell lines were SW1116, SAOS-2, HeLa, SK-OV-3, K562 and A375. ZBH-ZM-06 showed weak inhibitory activity against NCM460 cells.

ZBH-ZM-06 inhibited the relaxation of supercoiled DNA. To investigate whether ZBH-ZM-06 inhibits cell growth through the inhibition of Topo-1 activity, a DNA relaxation assay was performed in the presence of ZBH-ZM-06 or CPT-11 on supercoiled plasmid pHOT1 in vitro (Fig. 2). The results demonstrated that supercoiled DNA was relaxed by Topo-1 in the absence of drug (lane 2 of Fig. 2). In contrast, incubation with ZBH-ZM-06 or CPT-11 inhibited the relaxation of supercoiled DNA, as demonstrated by the increased intensity of the band corresponding to the supercoiled plasmid DNA. Furthermore, the inhibition of ZBH-ZM-06 occurred at a concentration as low as $1 \mu \mathrm{mol} / 1$, compared to CPT-11 (at $5 \mu \mathrm{mol} / \mathrm{l}$ ), indicating that ZBH-ZM-06 has a stronger inhibitory activity against Topo-1 than CPT-11.

Different patterns of tumor cell cycle arrest induced by ZBH-ZM-06, CPT-11 or SN38. The cell cycle arrest by ZBH-ZM-06, CPT-11 or SN38 at 24, 48 and $72 \mathrm{~h}$ was analyzed (Fig. 3). The cell cycle arresting patterns at the $S$ and $\mathrm{G}_{2} / \mathrm{M}$ phases induced by ZBH-ZM-06 were significantly different from those by CPT-11 or SN38 ( $<<0.0251-0.0001)$, except for K562 cells treated for $24 \mathrm{~h}$, which did not show a significant difference among the three groups. 
Table III. IC $_{50}$ values of ZBH-ZM-06, CPT-11 and SN38 in tumor cell lines and NCM460 cells.

\begin{tabular}{|c|c|c|c|c|c|c|}
\hline \multirow[b]{2}{*}{ No. } & \multirow[b]{2}{*}{ Cell line } & \multicolumn{3}{|c|}{$\mathrm{IC}_{50}(\mu \mathrm{mol} / \mathrm{l})$} & \multicolumn{2}{|c|}{ P-value } \\
\hline & & ZBH-ZM-06 & СРT-11 & SN38 & vs. CPT-11 & vs. SN38 \\
\hline 1 & SW1116 & $0.0679 \pm 0.0588$ & $2.7742 \pm 0.7676$ & $862.2826 \pm 151.3390$ & 0.0563 & 0.0045 \\
\hline 2 & SAOS-2 & $0.0000 \pm 0.0000$ & $0.3781 \pm 0.1157$ & $2.2989 \pm 0.5484$ & 0.0217 & 0.0002 \\
\hline 3 & A549 & $0.9331 \pm 0.9234$ & $3.7853 \pm 0.2473$ & $683.9418 \pm 255.6370$ & 0.2747 & 0.0072 \\
\hline 4 & SGC-7901 & $1.3539 \pm 0.4708$ & $19.5366 \pm 7.5020$ & $13.2479 \pm 6.2612$ & 0.0642 & 0.1331 \\
\hline 5 & 7860 & $0.0369 \pm 0.1414$ & $0.4515 \pm 0.1000$ & $4.9959 \pm 2.5830$ & 0.3400 & 0.0087 \\
\hline 6 & HeLa & $0.3025 \pm 0.1996$ & $68.4969 \pm 21.7217$ & $7630.3091 \pm 6997.9112$ & 0.0037 & 0.0054 \\
\hline 7 & SK-OV-3 & $0.0475 \pm 0.0404$ & $3.3985 \pm 1.2949$ & $88.4301 \pm 61.3723$ & 0.0256 & 0.0004 \\
\hline 8 & K562 & $0.0000 \pm 0.0000$ & $0.7551 \pm 0.3578$ & $1.2429 \pm 0.1687$ & 0.0298 & 0.0059 \\
\hline 9 & NCI-H446 & $0.3710 \pm 0.3924$ & $4.5701 \pm 4.6985$ & $26.0500 \pm 8.1501$ & 0.1056 & 0.0053 \\
\hline 10 & A375 & $0.0000 \pm 0.0000$ & $1.1970 \pm 0.7749$ & $7.3358 \pm 1.3535$ & 0.0169 & 0.0008 \\
\hline 11 & $\mathrm{MCF}-7$ & $5.3157 \pm 1.3016$ & $1.4336 \pm 0.3022$ & $3.3145 \pm 0.0007$ & 0.5764 & 0.1083 \\
\hline 12 & SMMC-7721 & $1.7157 \pm 2.2853$ & $12.2966 \pm 6.0580$ & $0.0028 \pm 0.0016$ & 0.7424 & 0.4478 \\
\hline 13 & NCM460 & $189.1652 \pm 49.3190$ & $31.8162 \pm 10.9849$ & $22.7732 \pm 6.1773$ & 0.8134 & 0.9053 \\
\hline
\end{tabular}

SW1116, colon adenocarcinoma; SAOS-2, human osteosarcoma; A549, non-small cell lung cancer; SGC-7901, gastric adenocarcinoma cancer; 7860, human renal carcinoma; HeLa, cervical carcinoma; SK-OV-3, ovarian cancer; K562, chronic myelogenous leukemia; NCIH446, non-small cell lung cancer; A375, human melanoma; MCF-7, breast cancer; SMMC-7721, hepatoma; NCM460, human colon mucosal epithelia.
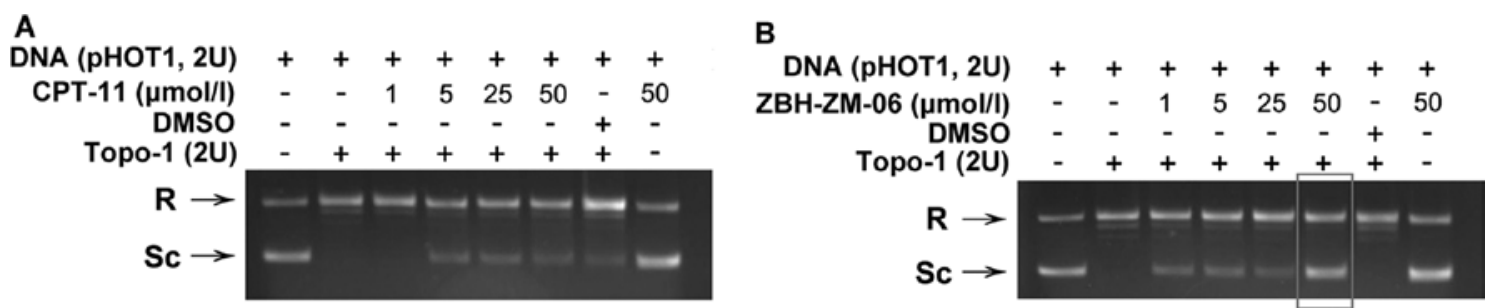

Figure 2. ZBH-ZM-06 and CPT-11 inhibited Topo-1-mediated supercoiled pHOT1-DNA relaxation. Closed loop pHOT1 plasmid DNA was incubated with the indicated concentration of CPT-11 (A) or ZBH-ZM-06 (B) (lanes 3-6) in the presence of Topo- 1 at $37^{\circ} \mathrm{C}$ for 30 min. Topo-1 alone (lane 2) was used as a positive control, and the absence of Topo-1 (lanes 1 and 8) was used as a negative control. Sc, supercoiled plasmid DNA; R, relaxed DNA. The assay was performed in triplicate.

Tumor cell apoptosis induced by ZBH-ZM-06. Tumor cell apoptosis induction by ZBH-ZM-06, CPT-11 and SN38 was analyzed by an Epics XL-MCL flow cytometer with FITC-Annexin V and PI staining. Fig. 4 shows the percentages of apoptotic SW1116 and SK-OV-3 cells induced by $10 \mu \mathrm{mol} / 1$ ZBH-ZM-06, CPT-11 and SN38 after $72 \mathrm{~h}$. The results indicated that ZBH-ZM-06 more efficiently induced apoptosis in SK-OV-3 and SW1116 cells either early (Annexin $\mathrm{V}^{+} / \mathrm{PI}^{-}$) or late (Annexin $\mathrm{V}^{+} / \mathrm{PI}^{+}$) than CPT-11 and SN38 $(\mathrm{P}<0.05)$. To test whether the operation process influences the results, we applied the non-adherent K562 cells to detect the number of apoptotic cells after $10 \mu \mathrm{mol} / 1 \mathrm{ZBH}-\mathrm{ZM}-06, \mathrm{CPT}-11$ and SN38 treatment for 24, 48 and 72 h (Fig. 5). As shown in Fig. 5, after $24 \mathrm{~h}$ of treatment, the number of apoptotic K562 cells in the ZBH-ZM-06 group was significantly higher than that in the CPT-11 and SN38 groups $(\mathrm{P}<0.05-0.0001)$. At this time, although cell cycle arrest had occurred (Fig. 3), there was no significant difference among the ZBH-ZM-06, CPT-11 and SN38 groups, indicating that cell apoptosis occurred earlier after drug treatment. This result is consistent with our previous conclusion (21). The number of apoptotic K562 cells treated with ZBH-ZM-06 for 48 and $72 \mathrm{~h}$ was significantly higher than that of cells treated with CPT-11 or SN38 $(\mathrm{P}<0.0001)$.

ZBH-ZM-06 promotes tumor cell apoptosis by activating caspase-3 and poly(ADP-ribose)polymerase (PARP). Caspase-3 is a key player in the execution of the apoptotic cascade by cleaving PARP. To determine whether ZBH-ZM-06 induced apoptosis through this pathway, the expression levels of cleaved capase- 3 and PARP were examined by western blot analysis. As shown in Fig. 6, ZBH-ZM-06 efficiently activated caspase- 3 to cleave PARP proteins.

The levels of the active form of caspase- 3 were determined by flow cytometry in the similarly treated groups as well. Consistent with the western blot results, the percentage of activated caspase- $3^{+}$K562, SK-OV-3 and SW1116 cells in the ZBH-ZM-06 group was higher than that of the CPT-11 and SN38 groups (Fig. 7). 

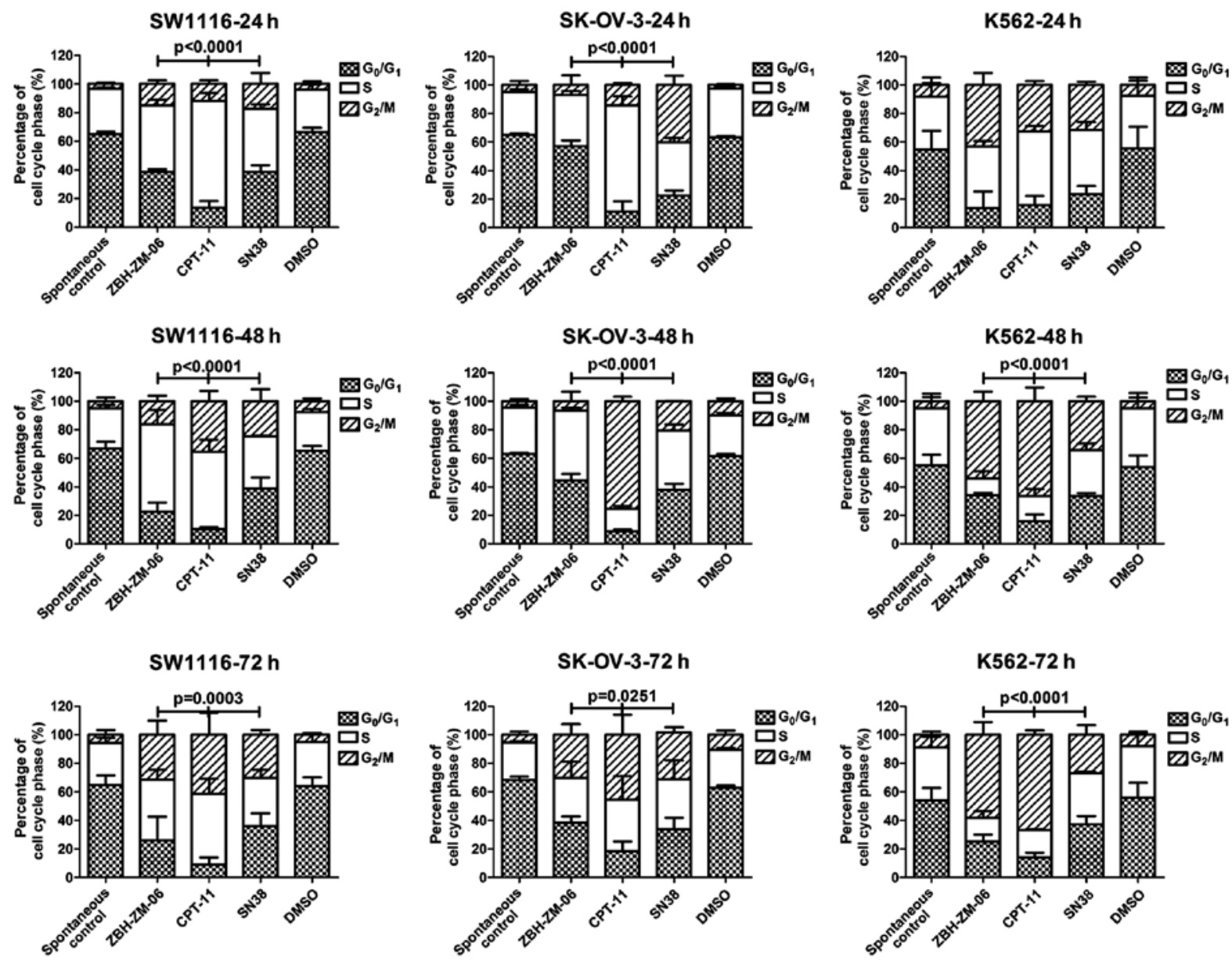

Figure 3. Tumor cell cycle arrest induced by ZBH-ZM-06, CPT-11, or SN38. The cell cycle arrest induced by ZBH-ZM-06, CPT-11 or SN38 was analyzed in SW1116 (left panels), SK-OV-3 (middle panels) and K562 (right panels) tumor cell lines. The distributions of $\mathrm{G}_{0} / \mathrm{G}_{1}$ (diagonal lines), $\mathrm{S}$ (open), and $\mathrm{G}_{2} / \mathrm{M}$ (dotted) phases were normalized to $100 \%$. Data are presented as the mean \pm standard deviation from three independent experiments.
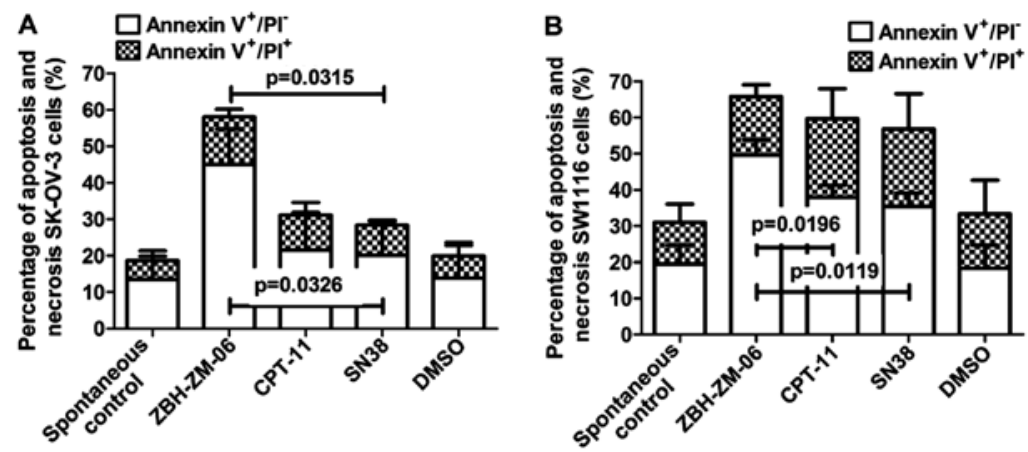

Figure 4. ZBH-ZM-06, CPT-11 and SN38 induced SK-OV-3 cell (A) and SW1116 cell (B) apoptosis. (A) SK-OV-3 cells and (B) SW1116 cells were treated with $10 \mu \mathrm{mol} / 1 \mathrm{ZBH}-\mathrm{ZM}-06$, CPT-11 and SN38, respectively, for $72 \mathrm{~h}$. The percentages of apoptotic cells were detected by an Epics XL/MCL flow cytometer with FITC-Annexin V and PI staining. The Annexin $\mathrm{V}^{+} / \mathrm{PI}^{-}$and Annexin $\mathrm{V}^{+} / \mathrm{PI}^{+}$cells indicated the early (dotted) and late (open) apoptosis, respectively. DMSO-treated cells were used as negative controls. Data are presented as the mean \pm standard deviation from three independent experiments. The statistical analysis of early apoptosis among the ZBH-ZM-06, CPT-11 and SN38 groups is presented above the bars, and that of late apoptosis is shown inside the bars.

\section{Discussion}

The present study reports the capacity of the novel CPT derivative ZBH-ZM-06 as a potential antitumor agent in vitro. ZBH-ZM-06 was created by reconstruction of SN38 to reduce several of the disadvantages of CPT-11, for example, poor solubility and causing severe diarrhea. SN38 is the active metabolite of CPT-11, which is $100-1000$ times more potent than CPT-11 (24). However, only a small portion (2-8\%) of it is finally converted to SN38. The variability of the conversion from CPT-11 to SN38 causes substantial dangerous toxicity risks and difficulties for the clinical management of the corresponding complications. Therefore, the direct administration of SN38 might be of benefit for cancer patients. However, the poor solubility in aqueous and other pharmaceutical solvents (including ethanol, cremophor and polysorbate 80 ) limits its 
A
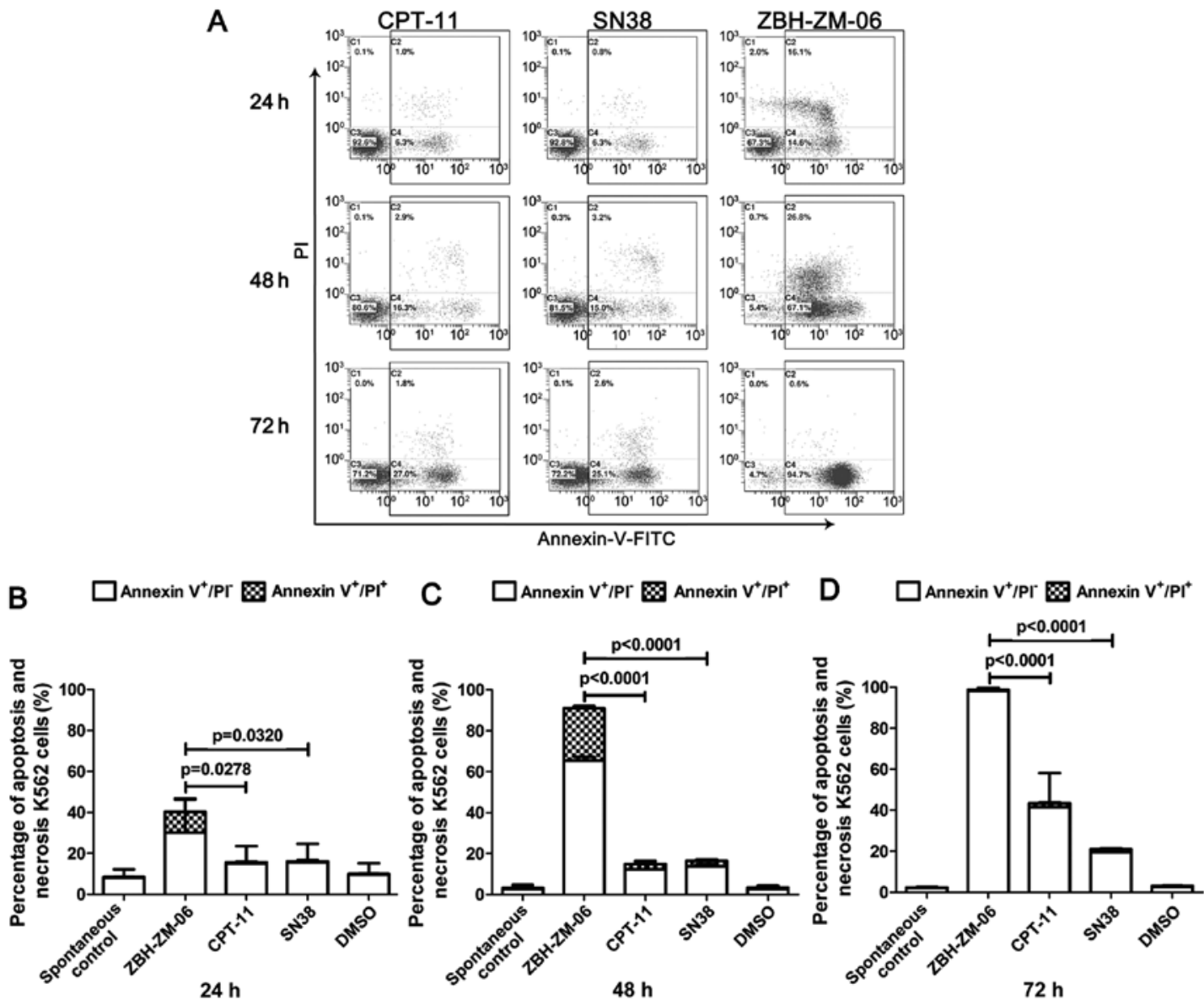

Figure 5. ZBH-ZM-06, CPT-11 and SN38 induce K562 cell apoptosis. K562 cells were treated with $10 \mu \mathrm{mol} / \mathrm{ZBH}-\mathrm{ZM}-06$, CPT-11 and SN38, respectively, for 24, 48 and $72 \mathrm{~h}$. The percentages of apoptotic cells were detected by an Epics XL/MCL flow cytometer with FITC-Annexin V and PI staining. The Annexin $\mathrm{V}^{+} / \mathrm{PI}^{-}$and Annexin $\mathrm{V}^{+} / \mathrm{PI}^{+}$cells indicated early (dotted) and late (open) apoptosis, respectively. DMSO-treated cells were used as negative controls. The typical biparametric images provided by the flow cytometer are presented in $\mathrm{A}$. The summarized data (mean \pm standard deviation from three independent experiments) are presented in B, $24 \mathrm{~h}, \mathrm{C}, 48 \mathrm{~h}, \mathrm{D}, 72 \mathrm{~h}$.

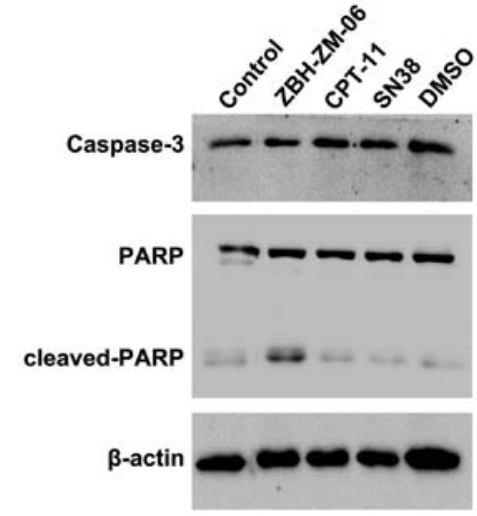

Figure 6. ZBH-ZM-06 promotes the activation of caspase-3 and PARP. SW1116 cells were treated with ZBH-ZM-06, CPT-11 and SN38 at $10 \mu \mathrm{mol} / 1$ for $72 \mathrm{~h}$. Non-treated (Control) and DMSO-treated cells were used as negative controls. Each lysate sample (50 $\mu \mathrm{g})$ was analyzed by western blot analysis with cleaved-caspase- 3 and cleaved-PARP antibodies. $\beta$-actin expression was detected on the same membrane as a protein loading reference. Experiments were repeated three times.

application (25), which has been proven previously by our group and others $(26,27)$.

Generally, CPT derivatives are expected to have improved solubility and stability. Yu et al (18) synthesized a series of 6-substituted indolizinoquinolinedione derivatives and evaluated them for their biochemical and biological activities. Zhou et al (28) evaluated the cytotoxicity of MXN-004 (a small-molecule compound of PEGylated SN38) in vitro and demonstrated that it has good water solubility. They further investigated the pharmacokinetics and tissue distribution of MXN-004 and its active metabolite SN38 in rats. We designed and synthesized the novel compound ZBH-ZM-06 by using a linear amino acid as a prodrug carrier. The amine of the amino acid was linked to the $10-\mathrm{OH}$ of SN38 via a carbamate linkage. $N$-methylpiperazine, which has good physiological compatibility, was linked to the C-terminus of the amino acid by an amide bond. The basic nitrogen atom of $N$-methylpiperazine can form a salt with the carboxyl group to improve the water solubility of the compound. One of the disadvantages of CPT-11 is that it is too stable under physiological conditions to be converted into the active form SN38, which results in a low efficiency. ZBH-ZM-06 overcame this disadvantage. It released SN38 rapidly and completely under physiological conditions ( $\mathrm{pH}$ 7.4), while remaining stable under acidic conditions ( $\mathrm{pH}$ 5.0). Therefore, it could be used as a lead compound for further drug development.

The anticancer activity of ZBH-ZM-06 was evaluated in 12 cancer cell lines representing diverse histologies. The results 
A
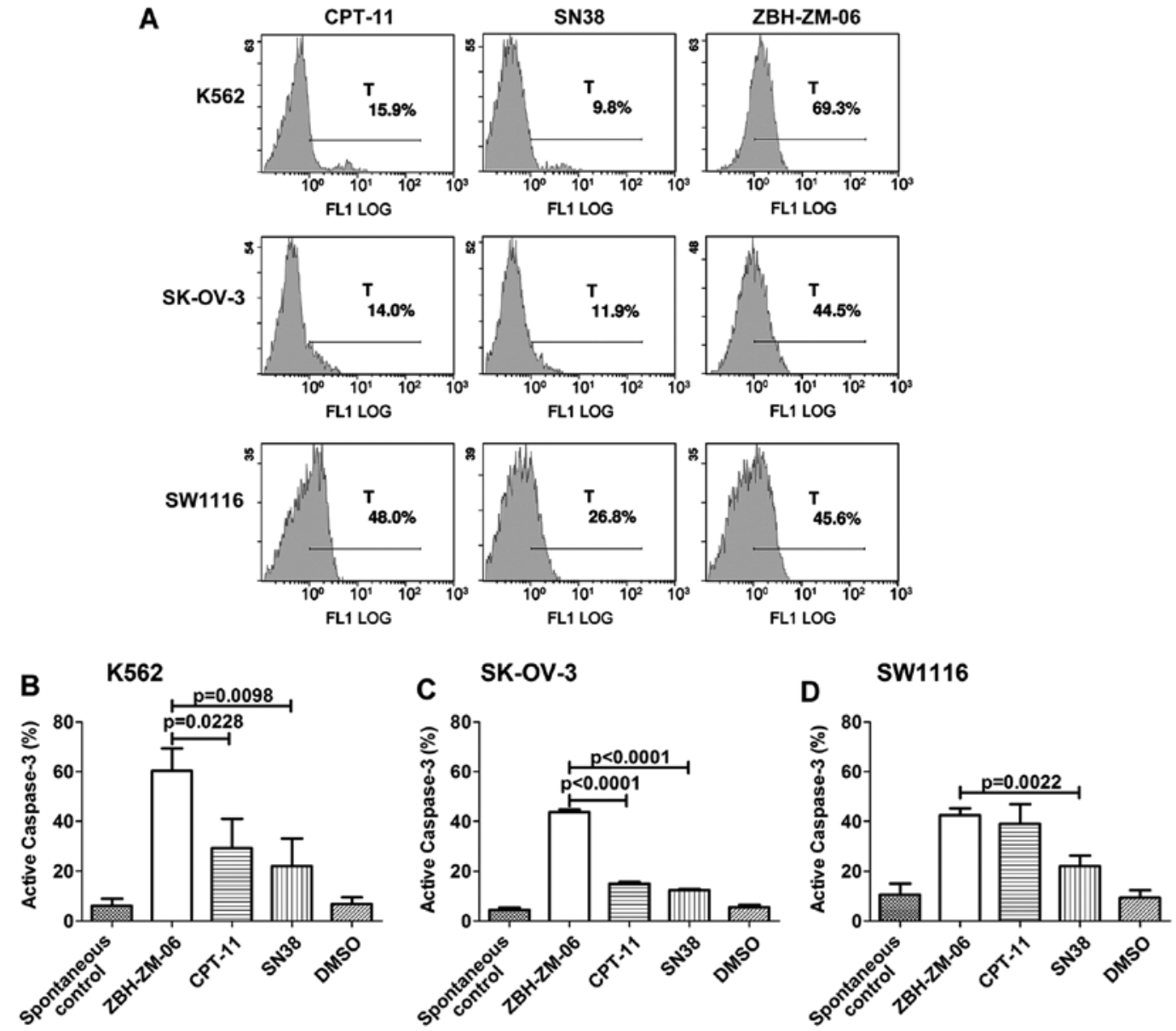

Figure 7. Increase of activated caspase-3 expression induced by ZBH-ZM-06, CPT-11 and SN38 in K562, SK-OV-3 and SW1116 cells. (A) Typical histograms of activated caspase- $3^{+}$cells by flow cytometry in the groups treated with $10 \mu \mathrm{mol} / 1 \mathrm{ZBH}-\mathrm{ZM}-06$ (right panels), CPT-11 (left panels), or SN38 (middle panels) in K562 (upper panels), SK-OV-3 (middle panels), and SW1116 (lower panels) cells. The quantitative analysis of activated caspase-3 in K562 (B), SK-OV-3 (C) and SW1116 (D) cells was performed from three independent experiments, respectively. Data are presented as the mean \pm standard deviation.

revealed that ZBH-ZM-06 had a stronger cytotoxic activity on nine cell lines (Table III). Such effects are comparable with those of previous studies. Demarquay et al (29) reported the characterization of BN80927, a novel CPT analog, and demonstrated that it was a very potent antiproliferative agent, as shown by the fact that the $\mathrm{IC}_{50}$ values were consistently lower than those of SN38 in tumor cell lines as well as in their related drugresistant lines. Lansiaux et al (26) determined whether an E-ring ketone derivative such as S36272 functions as a typical Topo-1 inhibitor, in a manner similar to TPT and SN38. The cytotoxicity of S38809 was studied on a panel of 34 cell lines, including 31 human tumor cell lines, P388 and P388CPT5 sublines, and normal pulmonary artery endothelial cells. S38809 proved to be a potent cytotoxic agent against the 31 human tumor cell lines, with a mean $\mathrm{IC}_{50}$ value of 5.4 vs. $11.6 \mathrm{nM}$ for TPT and $3.3 \mathrm{nM}$ for SN38; the colon, leukemia and ovary cell lines were relatively more sensitive. Consistent with our previous report, the correlation of the drug effect with the drug concentration was not considerable in ZBH-ZM-06-treated NCM460 cells, compared with CPT-11- or SN38-treated cells (21).

The 4-piperidinyl piperidine moiety of CPT-11 is responsible for inhibiting AchE activity, causing acute cholinergic diarrhea. This fact hinted to design a novel CPT derivative without AchE inhibition (30). ZBH-ZM-06 exhibited only weak inhibitory activity against AchE, compared to CPT-11, and showed that it can reduce acute cholinergic diarrhea associated with CPT-11. This result was comparable to those of previous studies $(31,32)$.

During DNA replication, Topo-1 primarily produces single-stranded breaks in DNA, which causes DNA relaxation to allow DNA replication. Once DNA replication is complete, Topo-1 will religate the single-stranded breaks to restore the double-stranded DNA structure. In order to explore the cytotoxic mechanism on DNA, we performed a conversional DNA relaxation assay to evaluate the inhibitory effect of ZBH-ZM-06 on Topo-1 activity. Topo-1-regulating drugs, such as CPT-11, can stabilize the covalent enzymeDNA complex to prevent DNA religation, thereby triggering a series of unstoppable DNA replications to induce cell death eventually (33). Compared to our previously reported structure (21) of ZBH-1205, the effective inhibitory concentration of ZHB-ZM-06 for DNA relaxation is $1 \mu \mathrm{mol} / \mathrm{l}$. The effective inhibitory concentration for DNA relaxation for ZBH-1205 is $50 \mu \mathrm{mol} / 1$. This DNA relaxation activity of ZHB-ZM-06 is even higher than that of CPT-11 (at $5 \mu \mathrm{mol} / \mathrm{l}$ ).

According to the cell cycle arrest analysis, our previously constructed compounds did not show an obvious effect on tumor cell cycle arrest, even though they induced apoptosis. In the present study, we modified the structure of ZBH-ZM-06 to improve the solubility and the stability, thus providing good 
effects. We treated SW1116, SK-OV-3 and K562 cells with ZBH-ZM-06 at a lower concentration $(10 \mu \mathrm{mol} / \mathrm{l})$ and analyzed the cell cycle at 24,48 and $72 \mathrm{~h}$ after treatment. The results suggested that ZBH-ZM-06 arrested the cell cycle of SW1116, SK-OV-3 and K562 cells at the $\mathrm{S}$ and $\mathrm{G}_{2} / \mathrm{M}$ phases (Fig. 3) at $24 \mathrm{~h}$ after treatment. With the extension of treatment, the S-phase arrest ratio was obviously increased and accompanied with a decrease of the $\mathrm{G}_{2} / \mathrm{M}$-phase arrest ratio. During the cell cycle in eukaryotes, the $\mathrm{G}_{2} / \mathrm{M}$ phase is a critical highly complex multi-stage process before cell division. Cell cycle arrest at the $\mathrm{G}_{2} / \mathrm{M}$ phase is a common cellular response to a variety of DNA-damaging agents. Topo-1 poisons, including CPT-11, induce replication-mediated double-stranded DNA breaks by a replication-fork collision mechanism and induce cell cycle arrest of cancer cells at the $\mathrm{G}_{2} / \mathrm{M}$ phase. Treatment of human colon cancer cells with SN38 also resulted in $\mathrm{G}_{2} / \mathrm{M}$ cell cycle arrest (34). Our results are consistent with previous reports, suggesting that the mechanism of ZBH-ZM-06 is similar to that of CPT-11 and SN38.

Cell cycle arrest allows time for the repair of DNA lesions. Cells re-enter the cell cycle if the damage can be adequately repaired, or they die by apoptosis if the damage is too severe. The apoptotic signal is transferred through a series of signaling cascades. Activation of cell surface receptors triggers the extrinsic signaling pathway to activate caspase- 8 , while cytochrome $c$ release from the mitochondria can initiate the intrinsic signaling pathway to subsequently induce caspase- 9 and caspase- 3 activation $(35,36)$. Caspase- 3 is a cysteine protease and a well-characterized effector of programmed cell death signaling. It is synthesized in normal cells as an inactive proenzyme that can be rapidly activated and transformed into cleaved caspase-3 (active form) by autoproteolytic cleavage or cleavage by other caspases. Cleaved caspase-3 allows caspase-activated DNase, also known as DNA fragmentation factor, to translocate to the nucleus where it is responsible for internucleosomal DNA cleavage, generating oligo-nucleosomal DNA fragments. Therefore, cleaved caspase- 3 is the executor in apoptosis signaling. During the process of apoptosis, caspase-3 cleaves the death substrate PARP to a specific $85-\mathrm{kDa}$ form.

A previous study has revealed that the pro-apoptotic activity of CPT-11 is via an intrinsic apoptotic signaling pathway (37). To validate whether ZBH-ZM-06 can trigger the intrinsic apoptotic signaling pathway, we detected activated caspase- 3 and cleaved PARP. Our investigation indicated that ZBH-ZM-06 increased the protein expression level of activated caspase- 3 and cleaved PARP, suggesting that similar to CPT-11, the pro-apoptotic activity of ZBH-ZM-06 is via an intrinsic signaling pathway.

According to proapoptotic activity analysis, the apoptosis of SW1116, SK-OV-3 and K562 cells induced by $10 \mu \mathrm{mol} / 1$ ZBH-ZM-06 for $72 \mathrm{~h}$ showed similar results as we reported previously. But the early apoptosis rate induced by ZBH-ZM-06 is higher than that induced by CPT-11 and SN38. Although there were some differences among these three tumor cell lines (Fig. 5A). Since the experimental procedures used for adherent cells may injure the cells, we analyzed K562 tumor cells in suspension at 24 and $48 \mathrm{~h}$. The results revealed that the rates of apoptosis and necrosis, especially the early apoptotic rate, induced by ZBH-ZM-06 for 24 and $48 \mathrm{~h}$ were significantly higher than those by CPT-11 and SN38 (Fig. 5B). These data suggested that the novel constructed CPT-11 derivative has a stronger proapoptotic capacity (38). Our results are in agreement with those reported by other research groups. Cao et al (39) explored apoptosis induced by SN38 and anti-Fas antibody (CH11) in WR/Fas-SMS1 cells and its possible mechanisms. The results indicated that the combination of SN38 and CH11 made the cells undergo greater apoptosis by finally activating caspase-3, whereas SN38 alone was ineffective at inducing these responses at any time-point. Di Francesco et al (40) also reported that Gimatecan (ST1481, LBQ707; 7-t-butoxyiminomethylcamptothecin), a novel lipophilic CPT derivative, was more cytotoxic than SN38 and TPT in a panel of neuroblastoma cell lines. Gimatecan's superior cytotoxicity is likely characterized by a marked arrest at the $\mathrm{G}_{2} / \mathrm{M}$ phase and induction of caspase 3-dependent apoptosis.

In conclusion, in this study, the CPT derivative ZBH-ZM-06 was designed and synthesized. It revealed higher water solubility and fewer side-effects than those of CPT-11 and SN38. Our results suggested that ZBH-ZM-06 had a greater ability to inhibit the viability of a broad spectrum of human tumor cells than CPT-11 and SN38, but it had less of an effect on normal cells. This antitumor activity may be obtained through Topo-1 inhibition and intrinsic apoptosis activation. This study provides experimental evidence that the novel CPT derivative is more effective and has fewer side-effects than other CPT derivatives. Therefore, it may be applied in the clinic for cancer treatment.

\section{Acknowledgements}

The present study was supported by funding provided by the Basic Research Project of Science and Technology Development in Jilin Province (to H.Y., grant no. 20130102097JC) and the Health Department Research Project of Jilin Province (to D.-W.Z., grant no. 2012Z037).

\section{References}

1. Wall ME, Wani MC, Cook CE, Palmer KH, McPhail AT and Sim GA: Plant antitumor agents. I. The isolation and structure of camptothecin, a novel alkaloidal leukemia and tumor inhibitor from camptotheca acuminata1,2. J Am Chem Soc 88: 3888-3890, 1966.

2. Lin RW, Yang CN, Ku S, Ho CJ, Huang SB, Yang MC, Chang HW, Lin CM, Hwang J, Chen YL, et al: CFS-1686 causes cell cycle arrest at intra-S phase by interference of interaction of topoisomerase 1 with DNA. PLoS One 9: e113832, 2014.

3. Tomicic MT and Kaina B: Topoisomerase degradation, DSB repair, p53 and IAPs in cancer cell resistance to camptothecinlike topoisomerase I inhibitors. Biochim Biophys Acta 1835: 11-27, 2013

4. Joerger M, Hess D, Delmonte A, Gallerani E, Fasolo A, Gianni L, Cresta S, Barbieri P, Pace S and Sessa C: Integrative population pharmacokinetic and pharmacodynamic dose finding approach of the new camptothecin compound namitecan (ST1968). Br J Clin Pharmacol 80: 128-138, 2015.

5. Joerger M, Hess D, Delmonte A, Gallerani E, Barbieri P, Pace S and Sessa C: Phase-I dose finding and pharmacokinetic study of the novel hydrophilic camptothecin ST-1968 (namitecan) in patients with solid tumors. Invest New Drugs 33: 472-479, 2015.

6. Naumczuk B, Kawęcki R, Bocian W, Bednarek E, Sitkowski J and Kozerski L: Preliminary study of mechanism of action of SN38 derivatives. Physicochemical data, evidence of interaction and alkylation of DNA octamer d(GCGATCGC $)_{2}$. Magn Reson Chem 55: 128-136, 2017.

7. Hamilton G, Klameth L, Rath B and Thalhammer T: Synergism of cyclin-dependent kinase inhibitors with camptothecin derivatives in small cell lung cancer cell lines. Molecules 19: 2077-2088, 2014. 
8. Vladu B, Woynarowski JM, Manikumar G, Wani MC, Wall ME, Von Hoff DD and Wadkins RM: 7- and 10-substituted camptothecins: Dependence of topoisomerase I-DNA cleavable complex formation and stability on the 7- and 10-substituents. Mol Pharmacol 57: 243-251, 2000.

9. Chazin EL, Reis RR, Junior WT, Moor LF and Vasconcelos TR: An overview on the development of new potentially active camptothecin analogs against cancer. Mini Rev Med Chem 14: 953-962, 2014

10. Zubovych IO, Sethi A, Kulkarni A, Tagal V and Roth MG: A novel inhibitor of topoisomerase I is selectively toxic for a subset of non-small cell lung cancer cell lines. Mol Cancer Ther 15: 23-36, 2016.

11. Liu YP, Chen HL, Tzeng CC, Lu PJ, Lo CW, Lee YC, Tseng CH, Chen YL and Yang CN: TCH-1030 targeting on topoisomerase I induces S-phase arrest, DNA fragmentation, and cell death of breast cancer cells. Breast Cancer Res Treat 138: 383-393, 2013.

12. Jeansonne DP, Koh GY, Zhang F, Kirk-Ballard H, Wolff L, Liu D, Eilertsen K and Liu Z: Paclitaxel-induced apoptosis is blocked by camptothecin in human breast and pancreatic cancer cells. Oncol Rep 25: 1473-1480, 2011.

13. Numbenjapon T, Wang J, Colcher D, Schluep T, Davis ME, Duringer J, Kretzner L, Yen Y, Forman SJ and Raubitschek A: Preclinical results of camptothecin-polymer conjugate (IT-101) in multiple human lymphoma xenograft models. Clin Cancer Res 15: 4365-4373, 2009.

14. Morandi E, Severini C, Quercioli D, D'Ario G, Perdichizzi S, Capri M, Farruggia G, Mascolo MG, Horn W, Vaccari M, et al: Gene expression time-series analysis of camptothecin effects in U87-MG and DBTRG-05 glioblastoma cell lines. Mol Cancer 7: 66, 2008.

15. Chen YL, Chueh FS, Yang JS, Hsueh SC, Lu CC, Chiang JH, Lee CS, Lu HF and Chung JG: Antitumor effects with apoptotic death in human promyelocytic leukemia HL-60 cells and suppression of leukemia xenograft tumor growth by irinotecan HCl. Environ Toxicol 30: 803-815, 2015.

16. Yao Y, Su X, Xie Y, Wang Y, Kang T, Gou L, Yi C and Yang J: Synthesis, characterization, and antitumor evaluation of the albumin-SN38 conjugate. Anticancer Drugs 24: 270-277, 2013.

17. Meco D, Di Francesco AM, Cusano G, Bucci F, Pierri F Patriarca V, Torella AR, Pisano C and Riccardi R: Preclinical evaluation of the novel 7-substituted camptothecin Namitecan (ST1968) in paediatric tumour models. Cancer Chemother Pharmacol 70: 811-822, 2012.

18. Yu LM, Zhang XR, Li XB, Yang Y, Wei HY, He XX, Gu LQ Huang ZS, Pommier Y and An LK: Synthesis and biological evaluation of 6-substituted indolizinoquinolinediones as catalytic DNA topoisomerase I inhibitors. Eur J Med Chem 101: 525-533, 2015.

19. Fukuda Y, Kanbe M, Watanabe M, Dan K, Matsuzaki K Kitanaka S and Miyata S: 3EZ,20Ac-ingenol, a catalytic inhibitor of topoisomerases, downregulates p-Akt and induces DSBs and apoptosis of DT40 cells. Arch Pharm Res 36: 1029-1038, 2013.

20. Zhou M, Liu M, He X, Yu H, Wu D, Yao Y, Fan S, Zhang P, Shi $\mathrm{W}$ and Zhong B: Synthesis and biological evaluation of novel 10-substituted-7-ethyl-10-hydroxycamptothecin (SN-38) prodrugs. Molecules 19: 19718-19731, 2014.

21. Wu D, Shi W, Zhao J, Wei Z, Chen Z, Zhao D, Lan S, Tai J, Zhong B and $\mathrm{Yu} \mathrm{H}$ : Assessment of the chemotherapeutic potential of a new camptothecin derivative, $\mathrm{ZBH}-1205$. Arch Biochem Biophys 604: 74-85, 2016.

22. Shoemaker RH: The NCI60 human tumour cell line anticancer drug screen. Nat Rev Cancer 6: 813-823, 2006.

23. Rivory LP, Bowles MR, Robert J and Pond SM: Conversion of irinotecan (CPT-11) to its active metabolite, 7-ethyl-10-hydroxycamptothecin (SN-38), by human liver carboxylesterase. Biochem Pharmacol 52: 1103-1111, 1996.

24. Senter PD, Beam KS, Mixan B and Wahl AF: Identification and activities of human carboxylesterases for the activation of CPT-11, a clinically approved anticancer drug. Bioconjug Chem 12: 1074-1080, 2001.

25. Atyabi F, Farkhondehfai A, Esmaeili F and Dinarvand R: Preparation of pegylated nano-liposomal formulation containing $\mathrm{SN}-38$ : In vitro characterization and in vivo biodistribution in mice. Acta Pharm 59: 133-144, 2009.
26. Lansiaux A, Léonce S, Kraus-Berthier L, Bal-Mahieu C, Mazinghien R, Didier S, David-Cordonnier MH, Hautefaye P, Lavielle G, Bailly C, et al: Novel stable camptothecin derivatives replacing the E-ring lactone by a ketone function are potent inhibitors of topoisomerase I and promising antitumor drugs. Mol Pharmacol 72: 311-319, 2007.

27. Liu H, Lu H, Liao L, Zhang X, Gong T and Zhang Z: Lipid nanoparticles loaded with 7-ethyl-10-hydroxycamptothecin-phospholipid complex: In vitro and in vivo studies. Drug Deliv 22: 701-709, 2015.

28. Zhou S, Li N, Wang X, Li C, Tian F, Ren S, Zhang Y, He Y, Qiu Z, Zhao D, et al: In vitro cytotoxicity, pharmacokinetics and tissue distribution in rats of MXN-004, a novel conjugate of polyethylene glycol and SN38. Xenobiotica 44: 562-569, 2014.

29. Demarquay D, Huchet M, Coulomb H, Lesueur-Ginot L, Lavergne O, Camara J, Kasprzyk PG, Prévost G and Bigg DC: BN80927: A novel homocamptothecin that inhibits proliferation of human tumor cells in vitro and in vivo. Cancer Res 64: 4942-4949, 2004

30. HyattJL, Tsurkan L, Morton CL, Yoon KJ,Harel M,Brumshtein B, Silman I, Sussman JL, Wadkins RM and Potter PM: Inhibition of acetylcholinesterase by the anticancer prodrug CPT-11. Chem Biol Interact 157-158: 247-252, 2005.

31. Dodds HM, Hanrahan J and Rivory LR: The inhibition of acetylcholinesterase by irinotecan and related camptothecins: Key structural properties and experimental variables. Anticancer Drug Des 16: 239-246, 2001.

32. Patnaik A, Papadopoulos KP, Tolcher AW, Beeram M, Urien S, Schaaf LJ, Tahiri S, Bekaii-Saab T, Lokiec FM, Rezaï K, et al: Phase I dose-escalation study of EZN-2208 (PEG-SN38), a novel conjugate of poly(ethylene) glycol and SN38, administered weekly in patients with advanced cancer. Cancer Chemother Pharmacol 71: 1499-1506, 2013.

33. Wadkins RM, Bearss D, Manikumar G, Wani MC, Wall ME and Von Hoff DD: Topoisomerase I-DNA complex stability induced by camptothecins and its role in drug activity. Curr Med Chem Anticancer Agents 4: 327-334, 2004.

34. Motwani M, Sirotnak FM, She Y, Commes T and Schwartz GK: Drg1, a novel target for modulating sensitivity to CPT-11 in colon cancer cells. Cancer Res 62: 3950-3955, 2002.

35. Liu YQ, Li WQ, Morris-Natschke SL, Qian K, Yang L, Zhu GX, $\mathrm{Wu} \mathrm{XB}$, Chen $\mathrm{AL}$, Zhang SY, Nan X, et al: Perspectives on biologically active camptothecin derivatives. Med Res Rev 35: 753-789, 2015.

36. Li J, Ouyang Y, Zhang X, Zhou W, Wang F, Huang Z, Wang X, Chen Y, Zhang $\mathrm{H}$ and Fu L: Effect of HM910, a novel camptothecin derivative, on the inhibition of multiple myeloma cell growth in vitro and in vivo. Am J Cancer Res 5: 1000-1016, 2015.

37. Ohmori T, Podack ER, Nishio K, Takahashi M, Miyahara Y, Takeda Y, Kubota N, Funayama Y, Ogasawara H, Ohira T, et al: Apoptosis of lung cancer cells caused by some anti-cancer agents (MMC, CPT-11, ADM) is inhibited by bcl-2. Biochem Biophys Res Commun 192: 30-36, 1993.

38. Zuco V, De Cesare M, Zaffaroni N, Lanzi C and Cassinelli G: PLK1 is a critical determinant of tumor cell sensitivity to CPT11 and its inhibition enhances the drug antitumor efficacy in squamous cell carcinoma models sensitive and resistant to camptothecins. Oncotarget 6: 8736-8749, 2015.

39. Cao Y, Jin ZX, Tong XP, Yue S, Sakai T, Kawanami T, Sawaki T, Miki M, Iwao H, Nakajima A, et al: Synergistic effects of topoisomerase I inhibitor, SN38, on Fas-mediated apoptosis. Anticancer Res 30: 3911-3917, 2010.

40. Di Francesco AM, Riccardi A, Barone G, Rutella S, Meco D, Frapolli R, Zucchetti M, D'Incalci M, Pisano C, Carminati P, et al: The novel lipophilic camptothecin analogue gimatecan is very active in vitro in human neuroblastoma: A comparative study with SN38 and topotecan. Biochem Pharmacol 70: $1125-1136,2005$ 